\title{
Estudio cinético de bacterias metanogénicas a diferentes temperaturas
}

\author{
Jorge Luis Ortiz Carrillo; ; Jarson Alexis Rodríguez Chona² . Ángela Maritza Cajiao
} Pedraza $^{3}$; Julio Isaac Maldonado Maldonado ${ }^{4}$.

\begin{abstract}
${ }^{1}$ Laboratorio Aguas Programa de Ingeniería Ambiental, Facultad de Ingenieras y Arquitectura. Universidad de Pamplona, Ciudadela Universitaria. Pamplona Norte de Santander, (Colombia.).

2 Programa de Ingeniería Ambiental Facultad de Ingenierías y Arquitectura Universidad de

Pamplona, Ciudadela Universitaria. Pamplona Norte de Santander, (Colombia).

${ }^{3}$ Programa de Microbiología. Facultad Ciencias Básicas. Docente (E) Cepario, Universidad de

Pamplona, Ciudadela Universitaria. Pamplona Norte de Santander, (Colombia).

${ }^{4}$ Programa de Ingeniería Ambiental, Facultad de Ingenieras y Arquitectura Universidad de

Pamplona, Ciudadela Universitaria. Pamplona Norte de Santander, (Colombia).
\end{abstract}

\section{Resumen}

En este estudio se empleó, un consorcio microbiano previamente aislado de la fase metanogénica de un filtro anaerobio de flujo ascendente separado en dos fases DI-FAFS. El proyecto tuvo por objeto analizar y describir el comportamiento cinético de Methanobacterium spp y Methanococcus spp respectivamente, empleando condiciones anaerobias y la variable de temperatura como principal punto de partida en el proceso. Para llevar a cabo el procedimiento respectivo se realizó un pre-inoculo de los microorganismos en estudio 18hrs antes, luego se tomó el volumen correspondiente y se llevó el inoculo al medio respectivo utilizado para el crecimiento, bajos condiciones estrictas de anaerobiosis; se evaluó a diferentes periodos de incubación bajo agitación $155 \mathrm{rpm}$, seguidamente se procedió a la medida de absorbancias tomando la lectura de D.O a $620 \mathrm{~nm}$, al mismo tiempo sembrando en agar para conteo respectivo y obtener UFC/ML.

Finalmente se realizó una curva de crecimiento para cada microorganismos a las tres diferentes temperaturas $\left(20^{\circ} \mathrm{C}, 27^{\circ} \mathrm{C}\right.$ y $\left.34^{\circ} \mathrm{C}\right)$, encontrando que tanto los resultados prácticos como teóricos sustentan que dicho consorcio microbiano en la fase metanogénica requiere una temperatura óptima de crecimiento, hallando así que el mejor comportamiento del crecimiento de Methanobacterium spp y Methanococcus spp se encuentra en las temperaturas de $27^{\circ} \mathrm{C}$ y $34^{\circ} \mathrm{C}$.

PALABRAS CLAVE: consorcios microbianos; cinética crecimiento, condiciones anaeróbicas.

\section{Abstract}

\section{Kinetic study of methanogenic bacteria at different temperatures}


40

In this study, a previously isolated from an anaerobic methanogenic phase upflow filter separated into two phases DI-FAFS microbial consortium was employed. The project was to analyze and describe the kinetic behavior of Methanobacterium spp and Methanococcus spp respectively, using anaerobic conditions and the variable temperature as the main starting point in the process. To carry out the respective procedure a pre-inoculum of microorganisms study was conducted in $18 \mathrm{hrs}$ before, then the corresponding volume was taken and respective inoculum was the medium used for growth, under strict anaerobic conditions; was evaluated at different periods of incubation under agitation $155 \mathrm{rpm}$, then it gave proce-measuring absorbance reading taking $O D$ at $620 \mathrm{~nm}$, while seeding in agar to obtain respective count and CFU / ML.

Finally a growth curve for each microorganisms at three different temperatures (20 ${ }^{\circ} \mathrm{C}, 27^{\circ} \mathrm{C}, 34^{\circ} \mathrm{C}$ ) was conducted, finding that both the practical and theoretical results support that the microbial consortium in the methanogenic phase requires optimal temperature growth and finding the best growth performance and Methanococcus spp Methanobacterium spp is at temperatures of $27^{\circ} \mathrm{C}$ and $34^{\circ}$ C.

KEYWORDS: microbial consortia; growth kinetics, anaerobic conditions.

*Para citar este artículo: Ortiz Carrillo Jorge Luis et al . Estudio cinético de bacterias metanogénicas a diferentes temperaturas. Revista Bistua.2016.14(1):39-48

+ Autor para el envió de correspondencia y la solicitud de las separatas: Ángela Maritza Cajiao Pedraza. Programa de Microbiología. Facultad Ciencias Básicas.Universidad de Pamplona, Ciudadela Universitaria. Pamplona Norte de Santander, (Colombia).

-email:angelacajiaoster@gmail.com 
41

\section{Introducción}

La curva del crecimiento microbiano representa la evolución del número de células viables presente en un cultivo microbiano líquido a lo largo del tiempo de estudio, teniéndose en cuenta la cantidad de células por mililitro de un cultivo, con un volumen y una cantidad de nutrientes limitada; en ella se diferencias cuatro fases, la fase de retraso (a veces llamada fase de latencia), la fase de crecimiento exponencial o logarítmico, la fase estacionaria y la fase de muerte celular.

De las cuatro fases de la curva de crecimiento, habitualmente la fase de crecimiento exponencial o logarítmico es la que presenta mayor interés por ser la fase en la que el incremento del número de microorganismos es máximo y la célula se encuentra en su máximo proceso metabólico. Durante esta fase el tiempo de generación (g) de los microorganismos (el tiempo que la población de microorganismo necesita para duplicar su número) se mantiene contante.

La conversión anaerobia de complejos orgánicos en dióxido de carbono y metano requiere de la actividad coordinada de diferentes grupos tróficos de poblaciones bacterianas. Tradicionalmente la degradación anaerobia ha sido considerada como un proceso que acepta la existencia de tres grandes grupos bacterianos: Las bacterias formadoras de ácidos (o acidogénicas), las formadoras de acetatos (o acetogénicas) y finalmente las formadoras de metano (o metanogénicas) (McCarty \& B.E., 2001) (C.P., Daigger, \& H.C., 1999).

En un proceso equilibrado, las bacterias metanogénicas durante el trascurso de las etapas de crecimiento generan a la misma velocidad: dióxido de carbono, metano, nitrógeno, hidrógeno y otros gases según la naturaleza del vertido. La acción coordinada de los grupos bacterianos provoca que el $\mathrm{pH}$ del medio se estabilice en valores ligeramente alcalinos, en el rango de 7.4 a 8.5 dependiendo de la temperatura de trabajo. (N. \& ., 1997).

Los cambios en las condiciones físicas o biológicas del medio implican cambios metabólicos y de adaptación de las distintas especies, alterando o modificando la actividad natural de las poblaciones implicadas. Así durante la fase de puesta en marcha del proceso anaerobio, las distintas familias microbianas evolucionan hasta que la biocenosis estabiliza todos los grupos alcanzando sus proporciones finales. (H.S. Shin, 2001).

La metanogénesis es el último paso del proceso de degradación anaerobia de la materia orgánica. (J. Andersson, 2002). En esta etapa, la mayor parte de la energía química contenida en el sustrato es convertida en metano por actuación de las bacterias metanogénicas. Este grupo bacteriano requiere condiciones medioambientales muy estrictas para su desarrollo y de elementos tales como: vitaminas, trazas minerales no usuales (como Ni y Co), AGV, cofactores específicos, etc. (McCarty \& B.E., 2001).

Para llevar acabo gráficamente este tipo de resultados se puede usar La edición web del DMFit que es una aplicación on-line utilizada para ajustar curvas bacterianas donde la fase lineal (fase exponencial) está precedida de una fase de adaptación y una posterior estacionaria. La edición web del DMFit ha sido elaborada con financiamiento del UK Food Standards Agency (Agencia de Normas Alimentarias del Reino Unido). (DMFit., 2009)

El objetivo general de este estudio fue analizar y describir el comportamiento
Comentado [AQ4]: Normas???????

Comentado [AQ5]: Citar???

Comentado [AQ6]: Lo mismo

\begin{tabular}{l}
\hline Comentado [AQ1]: \\
\hline Comentado [AQ2]: \\
Comentado [AQ3]: Citar: Normas Vancouver en todo el \\
texto \\
\hline Comentado [AQ7]: Normas
\end{tabular}


42

cinético del consorcio microbiano Methanobacterium spp y Methanococcus spp aislados previamente de un filtro anaerobio ascendente separado en dos fases, frente a la variable de temperatura y verificar su influencia en el mismo, para obtener así las características óptimas en las que se propone se deben estandarizar los procesos para que funcionen eficientemente.

\section{Materiales y Métodos}

Para estudiar la factibilidad de aplicación de un consorcio microbiano y para entender ¿cómo su eficiencia cinética es influenciada por las condiciones ambiéntales?, se empleó en este estudio un digestor anaerobio ascendente separado en tres fases en el rango mesófilos de temperaturas $\left(20^{\circ} \mathrm{C}, 27\right.$ ${ }^{\circ} \mathrm{C}$ y $34^{\circ}$ C) de manera discontinua bajo diferentes condiciones de operación. (Grupo de investigaciones GIMBIO programa de microbiología facultad de ciencias básicas Universidad de Pamplona (B. Demirel, 2002).

Para la inoculación inicial de la colonia microbiana se hizo necesario realizar el preinóculo de los microorganismos en estudios esto con el fin de favorecer su adaptación al medio y obtener una cepa joven en un intervalo de tiempo de incubación de 18$24 \mathrm{hrs}$ bajo condiciones estrictas de anaerobiosis, así que se requirió tomar valores de absorbancia del cultivo inoculado y del cultivo al término de la incubación, esto con el fin de observar el cambio en la biomasa que ocurre, producto del crecimiento del microorganismo.

\section{Inoculo}

Transcurrido este tiempo se tomó a partir del pre-inóculo un volumen de este, correspondiente al $0,25 \%$ del volumen del fermentador, de tal forma que se adicionaron a un Erlenmeyer de $500 \mathrm{ml}$ con $250 \mathrm{ml}$ de medio de enriquecimiento para bacterias metanógenas. (Atlas, 2010)

\section{Procedimiento experimental}

Una vez inoculado, se tomó una muestra de aproximadamente 1,5 $\mathrm{ml}$ (según el volumen de la cubeta de espectrofotometría) para medir inmediatamente la absorbancia y posteriormente incubado en la incubadora con agitación, a las temperaturas empleadas que para este caso fueron de $\left(20^{\circ} \mathrm{C}, 27^{\circ} \mathrm{C}\right.$ y $\left.34^{\circ} \mathrm{C}\right)$ y agitación (110 rpm) óptima del microorganismo bajo condiciones estrictas de anaerobiosis.

Las mediciones de absorbancia se realizaron cada 3, 6, 12 y 24 horas (dependiendo de las observaciones hechas), posteriormente las muestras obtenidas fueron inmediatamente procesadas, tomando la lectura de D.O a 620 $\mathrm{nm}$ y posteriormente sembradas por superficie en agar para conteo respectivo (Ortiz, 2008) para Methanobacterium spp y Methanococcus spp, esto con el fin de hacer un recuento en placa y así poder establecer valores de cinética, previamente estudiados que se reflejan en las Gráficas( $1,2,3,4,5)$

Para el recuento en placa, se sembró por la técnica en superficie en medio (Ortiz, 2008) selectivo, según el microorganismo trabajado, preparando diluciones consecutivas hasta $10^{-2}$ a partir de la muestra tomada del cultivo. Posteriormente, se procedió a aplicar las normas de conteo a los recuentos obtenidos, para establecer en cada muestra tomada la concentración UFC existente del microorganismo, y finalmente los valores obtenidos se grafican una función logarítmica: log del $N^{\circ}$ de células en función del tiempo, absorbancia vs tiempo, para cada tipo de microorganismos para cada temperatura empleada.

Por consiguiente se procede a establecer las gráficas que buscan expresar el comportamiento de los microorganismos
Comentado [AQ8]: Citar Nor Vancouver 
43

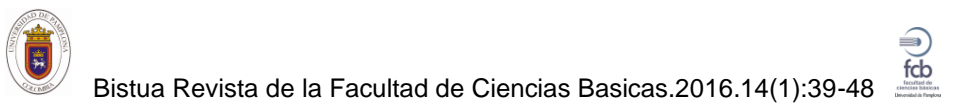

experimentados en los medios evaluados, constituyendo su cinética, de tal manera que se podrá estimar las fases de crecimiento: Latencia, Exponencial y entrada a la estacionaria, para esto se hizo necesario utilizar un software de modelamiento dinámico on-line DMFit. (DMFit., 2009)

\section{Resultados y Discusión}

Se empleó un consorcio microbiano de procedencia de un filtro anaerobio operado con estiércol porcino, agua residual y lixiviado, con la finalidad de establecer una cinética de reacción metabólica para cada microorganismo aislado dentro de la fase metano génica del sistema anaerobio. ( $R$. Borja, 2003).

Se reportan que existen varios mecanismos mediante los cuales se puede describir la oxidación de la materia orgánica mediante un consorcio microbiano. Mencionan que existen cuatro etapas bien definidas de reacción de acuerdo con los microorganismos presentes en el sistema, dándole a la etapa de la metanogenesis la mayor relevancia por ser la más prolongada en el tiempo y donde ocurren los cambios más notables de transformación.

Tabla 1. Valores de cinetica de crecimiento para Methanobacterium spp a $\mathrm{T}^{\circ} 20 \mathrm{C}$

\begin{tabular}{|c|c|c|c|c|c|c|c|c|}
\hline \multicolumn{9}{|c|}{ Nehaurobactenium spp } \\
\hline Tiempo & absorbancia & \multicolumn{4}{|c|}{ Dilución (recuento CFCmiL) } & Promedio & destiación & $\log X$ \\
\hline Hons & & $10^{-4}$ & $10^{4}$ & $10^{2}$ & $10^{2}$ & & & \\
\hline 0 & 0,123 & is & 98 & 90 & $n$ & 60,0205 & 42901539661 & 4,094606171 \\
\hline 3 & 0,127 & 180 & 177 & 100 & 100 & 93,3445 & 72,39663999 & 4336404075 \\
\hline 6 & 0,132 & 323 & 270 & 190 & 187 & 162,68066067 & 122,1531851 & $5,09183835.4$ \\
\hline 12 & 0,197 & 50 & 300 & 300 & 255 & 251,1993 & 199,316292 & 5,52624744 \\
\hline 24 & 0,198 & 588 & 490 & 333 & 278 & 285,533 & 218,1049963 & 5,6543597609 \\
\hline
\end{tabular}

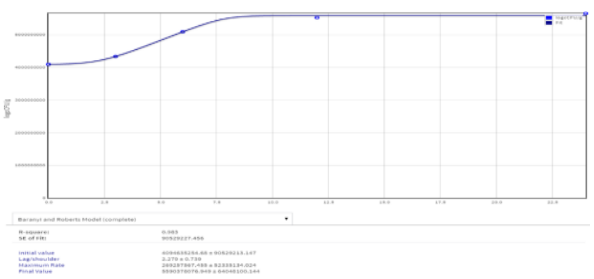

Gráfica 1. DMFit Curva de crecimiento para Methanobacterium spp a $\mathrm{T}^{\circ}$ de $20^{\circ} \mathrm{C}$ bajo parámetros del modelo experimental de Baranyi y Roberts (complete) (Baranyi \& Roberts, 1994)

Inicialmente se puede establecer según los datos obtenidos, se evidencian un crecimiento lento de Methanobacterium spp analizado bajo esta condición de temperatura mostrando así su mejor actividad metabólica trascurrida las $24 \mathrm{hrs}$, mostrando inicialmente una población de $4,07 \times 10^{9}$ una tasa máxima de crecimiento de $1,7 \times 10^{7} \mathrm{ucf} / \mathrm{ml}$, y con una población final de $5,6 \times 10^{9} \mathrm{ufc} / \mathrm{ml}$, lo que muestra que el factor temperatura muestra un límite donde las proteínas y ácidos nucleicos se pueden inactivar de manera reversible, y por ende a más bajas temperaturas este tipo de microorganismos limitan su crecimiento hasta su fase de muerte.

Para cada microorganismo existe una temperatura mínima por debajo de la cual no tiene lugar la proliferación o este se manifiesta en un crecimiento retardado como sucede para este caso.

Tabla 2. Valores de cinética de crecimiento para Methanococcus spp a T²0C

\begin{tabular}{|c|c|c|c|c|c|c|c|c|}
\hline \multicolumn{9}{|c|}{ Yedenococceas sp } \\
\hline Tiempo & absorbacin & \multicolumn{4}{|c|}{ Dilvöina (reveato LFC Mli) } & Promedio & desiacion & $\log \mathrm{X}$ \\
\hline Hors & & Wetet & 10ent & 10fet & 10 eto & & & \\
\hline l & (19 & 81 & $A{ }^{\circ}$ & 88 & $m$ & & & 0073 \\
\hline 3 & 0115 & 10 & 10 & Q & 8 & 691093323 & 4959311794 & 4) $3(032035$ \\
\hline 6 & 0116 & 20 & 19) & 127 & $\varphi$ & $102022660 ?$ & 773938868 & 455105011 \\
\hline 12 & 0221 & 58 & 48 & 30 & 233 & 2002035 & 242600338 & 5635316125 \\
\hline 4 & 0227 & 688 & 543 & 39 & 33 & 39,3711667 & 249,4661118 & 5,97185281 \\
\hline
\end{tabular}




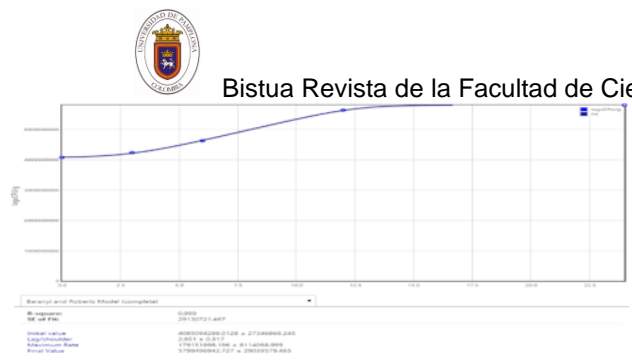

Gráfica 2. DMFit Curva de crecimiento para Methanococcus spp a $\mathrm{T}^{\circ}$ de $20^{\circ} \mathrm{C}$ bajo parámetros del modelo experimental de Baranyi y Roberts (complete) (Baranyi \& Roberts, 1994)

Igualmente para este microorganismo la temperatura es un factor que influye en su desarrollo metabólico haciendo de este un proceso aletargado, inicialmente partimos de una población inicial de 4,1×109 ufc/ml, manifestando una tasa de crecimiento de $1,8 \times 108 \mathrm{ufc} / \mathrm{ml}$ y una población final de $4,1 \times 109$ de acuerdo a los valores anteriores (Gráfica 2) esta bacteria tuvo un comportamiento un poco más significativo, mostrando su mayor punto de desarrollo metabólico en un periodo de incubación de $24 \mathrm{hrs}$ con referencia al crecimiento de Methanobacterium spp ( Gráfica 1). Este comportamiento sugiere que los organismos no están trabajando a la temperatura óptima.

Tabla 3. Valores de cinética de crecimiento para Methanobacterium spp a $\mathrm{T}^{\circ} 27^{\circ} \mathrm{C}$

\begin{tabular}{|c|c|c|c|c|c|c|c|c|}
\hline \multicolumn{9}{|c|}{ Mehanobucterium spp } \\
\hline Tiempo & absorbancia & \multicolumn{4}{|c|}{ 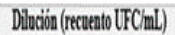 } & Promedio & Dessiación & $\log 1$ \\
\hline Horas & & $10^{-1}$ & $10^{4}$ & $10^{2}$ & $10^{2}$ & & & \\
\hline 0 & 0,168 & 300 & 360 & 200 & 215 & 200,8613333 & 149,0073078 & 5,3060617786 \\
\hline 3 & 0,18 & 400 & 300 & 315 & 290 & 221,6633333 & 159,019747 & 5,399005994 \\
\hline 6 & 0,19 & 530 & 400 & 40 & 30 & 286,032 & 211,2069312 & 5,666106093 \\
\hline 12 & $1,1,41$ & 600 & 510 & 420 & 388 & 335,1901660 & 250,1581499 & 3,8146980022 \\
\hline 24 & 1,200 & 62 & 588 & 488 & 300 & 346,688 & 253,662448 & 5,840257863 \\
\hline
\end{tabular}

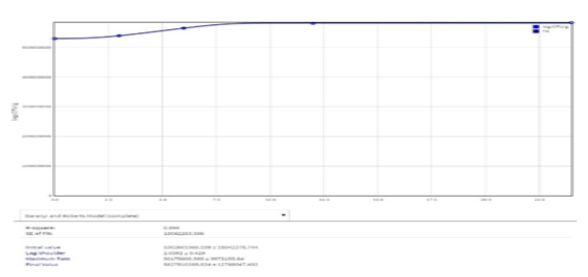

Gráfica 3. DMFit Curva de crecimiento para Methanobacterium spp a $T^{\circ}$ de $27^{\circ} \mathrm{C}$ bajo parámetros del

modelo experimental de Baranyi y Roberts (complete) (Baranyi \& Roberts, 1994).

Para este caso a medida que la temperatura aumenta, se incrementan también las reacciones enzimáticas y la tasa de reproducción por lo que al emplear este rango de temperatura cercana a su punto óptimo de crecimiento estas bacterias aceleran su metabolismo, la curva de crecimiento nos traza un comportamiento más simoidal; Partiendo de una población inicial de 5,3×109 ufc/ml, con una tasa máxima de crecimiento en su fase exponencial de $9 \times 109 \mathrm{ufc} / \mathrm{ml}$ llegando a una población final de 5,8x109, lo que muestra que ya la bacteria en estudio comienza a favorecerse para su proliferación debido a las condiciones de crecimiento dada.

Tabla 4 . Valores de cinetica de crecimiento para Methanococcus spp a $T^{\circ} 27 \mathrm{C}$

\begin{tabular}{|c|c|c|c|c|c|c|c|c|}
\hline \multicolumn{9}{|c|}{ Whiouococas yp } \\
\hline Tiempo & absortancia & \multicolumn{4}{|c|}{ Divcö́n (reveatio TFCmi) } & Promedio & Destación & $\log \int$ \\
\hline Hons & & IN & $\mathbb{W N}^{\mathrm{N}}$ & 15 & $1 \mathbb{1}^{4}$ & & & \\
\hline$T$ & 0,168 & 300 & 360 & 30 & 30 & 236,69646067 & 162304536 & $5,4364023]$ \\
\hline 3 & 0,17 & 438 & 362 & 315 & 298 & $2360285 \% 5$ & 1715YSW\$\% & 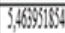 \\
\hline 6 & 0,206 & 70 & 610 & 525 & 40 & 30,534353 & 233,267665 & 5 S56301:2 \\
\hline 12 & 1225 & 876 & 780 & 60 & 510 & 4465375 & $324+123366$ & $6.159394+13$ \\
\hline 24 & 1243 & 1000 & 90 & 70 & 630 & 50,2071667 & 39,497328 & 63047572 \\
\hline
\end{tabular}

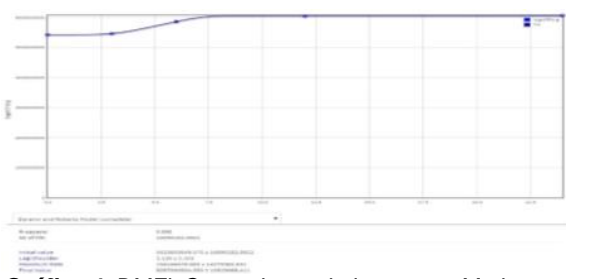

Gráfica 4. DMFit Curva de crecimiento para Methanococcus spp a $T^{\circ}$ de $27^{\circ} \mathrm{C}$ bajo parámetros del modelo experimental de Baranyi y Roberts (complete) (Baranyi \& Roberts, 1994)

Como el anterior caso esta bacteria comienza su actividad metabólica ya un poco más acelerada, con una mayor proliferación que Methanobacterium spp (Gráfica 3) partiendo con una población inicial de 5,4×1010, 
45 Bistua Revista de la Facultad de Ciencias Basicas.2016.14(1):39-48

mostrando una tasa máxima de crecimiento de $1,5 \times 108$ y llegando a una población final de $6,0 \times 109$, lo que coloca a Methanococcus spp en mejores términos de adaptación y crecimiento en este tipo de procesos anaerobios como mejor alternativa en la fase Metanogénica.

Tabla 5. Valores de cinética de crecimiento para Methanobacterium spp a $\mathrm{T}^{\circ} 34 \mathrm{C}$

\begin{tabular}{|c|c|c|c|c|c|c|c|c|}
\hline \multicolumn{9}{|c|}{ Methanobacterium spp } \\
\hline Tiempo & absorbascia & & Hechia (re & eato TTC & & Promedio & destiacion & \\
\hline Horss & & $10^{4}$ & $10^{4}$ & $10^{4}$ & $10^{2}$ & & & \\
\hline 1 & 0,185 & 860 & 600 & 740 & 460 & 665 & 149,916 & \\
\hline 3 & 0,236 & 1000 & 1000 & 980 & 930 & 977,5 & 28,013 & \\
\hline 6 & 0.592 & 17000 & 10000 & 17200 & 15000 & 16500 & 87,496 & \\
\hline 12 & 1,366 & 530000 & 29000 & 1600000 & 1250000 & 919500 & 526049,836 & 1 \\
\hline 24 & 1,992 & 970000 & 784000 & 1070000 & 980000 & 951000 & 103985,576 & 1 \\
\hline
\end{tabular}

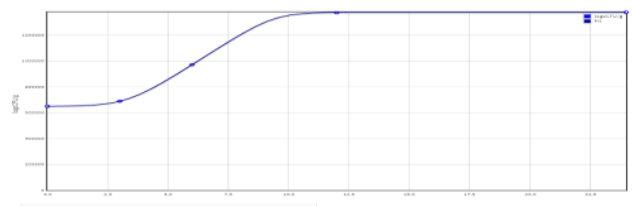

Gráfica 5. DMFit Curva de crecimiento para Methanobacterium spp a $\mathrm{T}^{\circ}$ de $34^{\circ} \mathrm{C}$ bajo parámetros del modelo experimental de Baranyi y Roberts (complete) (Baranyi \& Roberts, 1994)

Aun cuando la biota anaerobia puede crecer en un amplio rango de temperaturas, en este estudio se ha realizado la cinética a tres valores de temperaturas diferentes dentro del rango mesófilo. Como se sabe, ésta es una de las variables ambientales primordiales, ya que dicta los límites de viabilidad del proceso pues afecta a todos los microorganismos de manera diferente llegando incluso a lesionar la integridad celular de la población microbiana.

En la gráfica 5 se puede observar que la actividad del consorcio a esta temperatura está en su punto óptimo de crecimiento Por otro lado, el perfil mostrado por la gráfica 5, exhibió durante el periodo de incubación de $24 \mathrm{hrs}$ un proceder muy semejante que para el caso posterior en la curva 6 . No obstante, su comportamiento es en general el más activo de las cinéticas aquí analizadas ya que pese haber mostrado en pocos días de actividad, su número poblacional de microorganismos resultaron ser las más importantes de todos los ensayos realizados, llegando a ser al final de $14 \times 10^{4} \mathrm{UFC} / \mathrm{ml}$, que demuestra un nivel efectivo de este microorganismo en la fase metanogénica que conlleva a una muy buena biodegradación de la materia al interior de este tipo de procesos.

Tabla 6. Valores de cinética de crecimiento para Methanococcus spp a $\mathrm{T}^{\circ}$ de $34^{\circ} \mathrm{C}$

\begin{tabular}{|c|c|c|c|c|c|c|c|c|}
\hline \multicolumn{9}{|c|}{ Yethorococecas spp } \\
\hline Tiempo & absorbancin & \multicolumn{4}{|c|}{ Diluciba (recoento CFC/mL) } & Promedio & destiación & $\log \mathrm{N}$ \\
\hline Horas & & $10^{-1}$ & $10^{-1}$ & $10^{2}$ & $10^{2}$ & & & \\
\hline 0 & 0,152 & 655 & 40 & 620 & 500 & 573,75 & 81,652 & 635219 \\
\hline 3 & 0,666 & 1250 & 1120 & 1370 & 1190 & 1232,5 & 91,753 & 7,11679 \\
\hline 6 & 0,778 & 2800 & 2650 & 2900 & 3000 & 2837,5 & 12,301 & 795067 \\
\hline 12 & 0,933 & 45000 & 47000 & 41100 & 41000 & 43525 & 2574,271 & 10,6810 \\
\hline 24 & 0.993 & 111770 & 160000 & 98000 & 100000 & 11743 & 25127,523 & 11.6737 \\
\hline
\end{tabular}

Gráfica 6. DMFit Curva de crecimiento para Methanococcus spp a $\mathrm{T}^{\circ}$ de $34^{\circ} \mathrm{C}$ bajo parámetros del modelo experimental de Baranyi y Roberts (complete) (Baranyi \& Roberts, 1994)

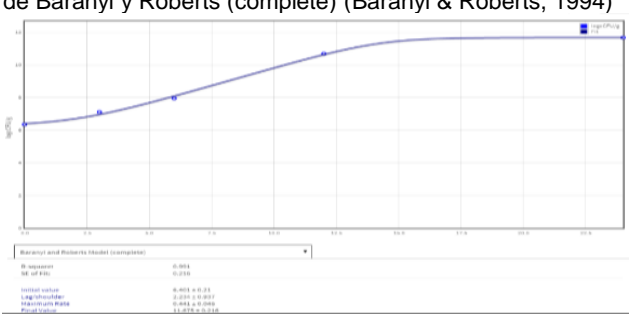

Los efectos de la temperatura mesofilica para el proceso cinético durante la fase metanogénica de la digestión anaerobia, conduce a que las reacciones metabólicas referente al crecimiento de dichos consorcios microbianos experimentados a temperaturas comprendidas entre $27^{\circ} \mathrm{C}$ y $34^{\circ} \mathrm{C}$, muestran una alta tasa de crecimiento poblacional como lo muestran las (Gráficas 3-4 y 5-6) en comparación con los operados a temperaturas bajas como la fue la de $20^{\circ} \mathrm{C}$, donde muestran una tendencia en cuanto a la multiplicación de este tipo de 
46

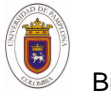

microorganismos bastante lenta, (Gráficas 1 -

2) con lo anterior se puede concluir que tanto

la temperatura como las condiciones estrictas de anaerobiosis, juegan un papel fundamental en este tipo de bacterias, en cuanto a la activación metabólica de estos se refiere, haciendo de estos la temperatura como una de la variables más eficaz para tener en cuenta en este tipo de procesos anaerobios, si se quiere conseguir una producción optima de la biomasa presente en esta etapa. (DMFit., 2009)

Antes de incorporar modelos de crecimiento microbiano en evaluaciones cuantitativas se debe considerar la validez de las investigaciones, ya que no todos los modelos de crecimiento microbiano están validados bajo condiciones reales, por lo que resultaría incorrecto suponer que las predicciones del modelo son correctas, si los puntos estimados son reemplazados por distribuciones de probabilidad en los parámetros que constituyen las variables de entrada del modelo. (G.C. Cha, 1997).

\section{Conclusiones}

Una vez activado el consorcio microbiano, anteriormente aislado y conservado, se procedió a la adaptación al sustrato; los resultados conseguidos en la elaboración de la cinética de crecimiento para cada microorganismo confirman que para llegar a obtener un desarrollo metabólico óptimo, con este tipo de bacterias presente en la fase Metanogénica, la biomasa requiere de temperaturas mesofilas cercana a la óptima de $27^{\circ} \mathrm{C}$ a $34^{\circ} \mathrm{C}$ y por lo menos 24 horas incubación bajo condiciones estrictas de anaerobiosis. No obstante, debido a que se trata de una comunidad entrelazada de microorganismos, resulta difícil separar la actividad biológica de cada una de las fases.

En definitiva, como lo propone la literatura el cambio en $T^{\circ}$ puede actuar como un inhibidor del proceso biológico, ya que cuando éste no cinética de reacción es menos eficiente.

Este estudio, ha podido indicar que cambiar en 7 unidades el valor de la $\mathrm{T}^{\circ}$ origina incrementos de hasta el $50 \%$ en la eficiencia del crecimiento de este tipo de bacterias, por lo cual se debe tener precaución al manejar este tipo de variable.

Se puede sugerir que la manera óptima de trabajar éste sistema de reacción de ensayos de biocinéticas sería a $T^{\circ}$ de $34^{\circ} \mathrm{C}$ y $37^{\circ} \mathrm{C}$ con un periodo mínimo de incubación de $24 \mathrm{hrs}$.

Las temperaturas de operación analizadas manifiestan que el consorcio es muy perceptivo a los cambios de esta variable, y se obtuvo como temperatura óptima los $34^{\circ}$ C.

\section{Agradecimientos:}

Al programa de Microbiología de la Universidad de Pamplona por el apoyo. Se agradece la participación de M.Sc Danny Armando Pisciotti Ortega en el manejo del software DMFit para el comportamiento cinético microbiológico.

Referencias bibliogràficas

Atlas, R. M. (2010). Handbook of Comentado [AQ9]: Referenciar de acuerdo a las normas Vancouves
Microbiological Media. Washington D,C: ASM-PRESS.

B. Demirel, O. Y. (2002). Two-phase anaerobic digestion processes: a review, J. Chem. Technol. Biotechnol. 77, 743755.

Baranyi, J., \& Roberts, T. (1994). A dynamic approach to predicting bacterial growth in food. . Int. J. Food Microbiol.23: 277-294.

C.P., G., Daigger, G., \& H.C., L. (1999). Biological wastewater treatment. New York,: Marcel Dakker. 


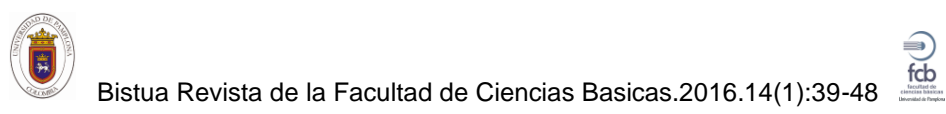

Prieto, E. D. J. M., Rivas, B., \& Sánchez, J. 2013. Natural polymer grafted with syntethic monomer by microwave for water treatment-a review. Ciencia en Desarrollo, 4(1): 219-240.

DMFit. (2009). Software de modelamiento dinámico edición online.http://ifrsvwwwdev.ifrn.bbsrc.ac.u k/CombasePMP/GP/DMFit.aspx.

G.C. Cha, T. N. (1997). Effect of rapid temperature change and HRT on anaerobic acidogenesis. Water Sci. Technol. , 247-253.

Moreno, L. M., Muñoz Prieto, E., \& Casanova, H. (2015). Flocculatin with Chitosan of Microalgae Native of the Colombian Plateau. Ciencia en Desarrollo, 6(1), 1724.

A Daza, F Herrera, A Naranjo (2013). Condiciones higiénico-sanitarias aplicadas en la elaboración de queso doble crema manufacturado en tres empresas de la provincia de Pamplona. Revista Bistua, Vol. 11, no.1, 61-73.

Gavala, H. N., Angelidaki, I., \& K., B. (2003). Kinetics and modeling of anaerobic digestion process. . Adv. In Bioch. Eng. \& Biotech., Vol. 81, pp. 58-93.

H.S. Shin, S. H. (2001). Performance of an $U A S B$ reactor treating leachate from acidogenic fermenter in the two-phase anaerobic digestion of food waste, Water Res. 35 (14) (2001) 3441-3447.

J. Andersson, L. B. (2002). Evaluation of straw as a biofilm carrier in the methanogenic stage of two-stage anaerobic digestion of crop residues. Bioresour. Technol. 85 (2002) 51-56.

McCarty, P., \& B.E., R. (2001). Environmental biotechnology: Principles and applications. Ed. McGraw Mil.Cap. 2, pp. 19-55.
Ortiz, J. F. (2008). Practica Laboratorio Microbiología Industrial: Crecimiento Bacteriano en Cultivo Discontinuo, Curva de Crecimiento. Pamplona, Colombia.

N., H. P., \& ., S. C. (1997). Rumen microbial ecosystem,. Ed. Elsevier applied Science, Cap 1. 117-199, (1997).

R. Borja, B. R. (2003). Kinetics of mesophilic anaerobic digestion of the two-phase olive mill solid waste. Biochem. Eng. J. 15 (2003) 139-145.

Jorge Luis Ortiz Carrillo Microbiólogo,
Laboratorio Aguas Programa de
Ingeniería Ambiental, Facultad de
Ingenieras y Arquitectura. Universidad de
Pamplona, Ciudadela Universitaria.
$\begin{aligned} & \text { Pamplona Norte de Santander, } \\ & \text { (Colombia.) }\end{aligned}$

Jarson Alexis Rodríguez Chona Maestría en Ingeniería Ambiental Facultad de Ingenierías y Arquitectura Universidad de Pamplona, Ciudadela Universitaria.

Ángela Maritza Cajiao Pedraza.M. Sc Docente, (E) Cepario, Programa de Microbiología. Facultad Ciencias Básicas. Universidad de Pamplona, Ciudadela Universitaria. Pamplona Norte de Santander, (Colombia).

Julio Isaac Maldonado Maldonado. M.Sc. Docente, Programa de Ingeniería Ambiental, Facultad de Ingenieras $y$ Arquitectura Universidad de Pamplona, Ciudadela Universitaria. Pamplona Norte de Santander, (Colombia) 